\title{
Mathematical model and technology to provide new resources of groundwater for irrigations
}

\author{
E. Nițescu ${ }^{1}$, I. Moruz ${ }^{1}$, C. Nițescu ${ }^{2}$, E. Chiorescu ${ }^{3} \&$ Şt. Popescu ${ }^{3}$ \\ ${ }^{\prime}$ Department of Cadastre, Technical University "Gh. Asachi", \\ Iassy, Romania \\ ${ }^{2}$ Department of Mathematics, Technical University "Gh. Asachi", \\ Iassy, Romania \\ ${ }^{3}$ Department of Hydroamelioration and Environment Protection, \\ Technical University "Gh. Asachi”, Iassy, Romania
}

\begin{abstract}
The opening and use of local sources of groundwater for irrigation offers important economic and social advantages. However, an extended use of groundwater is limited by the currently utilized technology for the tapping of these waters, which is very expensive. This paper shows a mathematical model and technological solution that allows the creation of local underground water accumulations at affordable prices for agricultural farms. Specifically for Romania is the fact that there is used mainly surface water (ca. 93\% of the total consumption). With a few exceptions, the groundwater is used only for the water supply of some urban areas. Today in Romania the reasons that make it difficult to introduce irrigation in agricultural farms or mini-farms are: a) the lack of water (in farms located outside irrigated areas); b) a prohibitive price for water delivered to farms situated at the boundaries of large irrigation systems; c) costs generated by the huge transport distances of the water, by re-pumping and by water losses that sometimes exceed $40 \%$ of the volume carried via channel network. These considerations show evidently that, in order to deliver water to also farms located far away from surface-water sources, the only economic solution is the use of local groundwater resources. The primary data from field and/or lab studies have to be systematized and analysed on various categories: topographic, climatic, hydrologic, hydro-geologic, pedological, geological and chemical. Moreover, there have been set the basic work assumptions, all these leading to the construction of the conceptual and the mathematical models for underground reservoirs' design. Taking into account the fact that in Romania such irrigation systems have not been built and the prices for conventional technologies are prohibitive, at least today, we propose a new technology, based on a Patent Ro 94257 Nitescu (Installation for the Execution of Vertical, Nonpermeable Screens, 1987). This technology has to be mainly focused on a reducing of the specific investment costs, because the lowering of costs becomes a crucial condition for a sustained development of agriculture.

Keywords: groundwater, underground water accumulations, technological solution, conceptual and mathematical models, irrigation.
\end{abstract}




\section{Introduction}

Romania is characterized by the fact that there is used mainly surface water (ca. $93 \%$ of the total consumption). The groundwater, with a few exceptions, is used only for the water supply of some urban areas. In developed countries the system is better balanced, in France, for example Niţescu et al [3], groundwater represents $60 \%$ of the consumption, agriculture using $68 \%$ of the total water, whilst industries use only $5 \%$ (due to the general implementation of water recycling).

Today in Romania the reasons that make it difficult to introduce irrigations in agricultural farms or mini-farms are: a) the lack of water (in farms located outside irrigated areas); b) a prohibitive price for water delivered to farms situated at the boundaries of large irrigation systems; c) costs generated by the huge transport distances of the water, by re-pumping and by water losses that exceed sometimes $40 \%$ of the volumes carried via channel network.

These considerations show evidently that, in order to deliver water also to farms located far away from surface-water sources, the only economic solution is a more extensive use of local groundwater resources.

However, in many agricultural areas of Romania, the groundwater and deeper aquifers do not provide flows complying with the real needs. Hence, it is mandatory to create local reservoirs of groundwater (at relatively small depths), action which can be justified by the following arguments: a) the use of groundwater for irrigations provides better conditions in terms of seasonal, annual and multi-annual adjustment of irrigation systems; b) for agricultural surfaces on that prevails a salt-ascension risk, the use of groundwater eliminates the need of desalination draining systems; c) in agricultural areas with a high groundwater level, the tapping of groundwater lowers this level, fact that is benefit for cultures; d) the water losses generated by evaporation are eliminated.

Therefore a rational use of the upper aquifer does not harm the environment through negative hydro-geological alterations. Furthermore it helps to keep the balance of water within irrigated areas, by decreasing the risk of soil deterioration and of secondary salt marshes. Along the same line, in India there is a law that obliges farmers to use local groundwater for irrigations, providing that in the agricultural areas there is an adequate groundwater level. In terms of environment (soil's) protection some objections will arise about the groundwater's mineral content, which frequently is higher than that of surface waters. The answer to provide for this issue is that in the case of an underground reservoir, the water which is continuously consumed and replaced will progressively decrease its load of minerals. In these situations a solution is represented by performing a dilution with non-mineralized water until the load of minerals gets to admissible limits, or to provide irrigation only on light soils [3].

\section{Agricultural areas fitted for the creation of local underground reservoirs from upper-aquifer waters}

The areas in that can be conceived underground water reservoirs are: 
a) Areas located at the base of terraces, if within these exists a permanent water flow.

b) Wetland areas; studies proved that the groundwater afflux in rivers represent $10-40 \%$ of the river's total flow. Mainly, the feeding of the river from aquifers takes place during the low water period. Considering that the water deficit for irrigation appears during a low water period, it results that the local underground reservoir has to be designed in order to avoid water losses towards the river bed in the area of the aquifer tapping. In these conditions, the feeding of the reservoir is not made also from river, but only from infiltrated rain waters and from the drainage of the underground hydrographical basin of the designed reservoir. Exceptions are the greater rivers with slight level fluctuations, the feeding of the aquifer from the river being quasi-permanent (e.g. the Danube River's wetland).

c) Irrigated areas where the groundwater level has considerably increased due to water losses in the distribution system.

d) Areas in that the aquifer can be artificially loaded.

In order to tap water from the underground reservoir, can be employed one of the following usual technical solutions: $1^{\circ}$ tapping shafts, if the groundwater layer is mighty and assures a sufficient afflux; $2^{\circ}$ shafts with radial drain pipes, if the groundwater has a small flow rate; $3^{\circ}$ horizontal catching galleries, solution that can be applied only when the aquifer is at a small depth (max.10-15 m).

In Romania farms needing irrigations are located in plateau or plain areas, where the groundwater assures in most cases only small rates of flow. This situation would require solutions of the type $2^{\circ}$ or $3^{\circ}$, both implying prohibitive costs for a farm or even for farm associations.

The conclusion is that, depending on hydrological and hydro-geological conditions, the local underground reservoirs can supply irrigation systems. The water of the reservoir can be used alternatively to complement a surface source during peak consumption periods, or to provide the whole irrigation water volumes at pre-defined flow rates. Outside irrigation periods the available groundwater can be used for other activities, which will contribute to a faster investment amortization. A complex use of water stored in underground reservoirs allows also a good control of groundwater layers in order to avoid an excessive humidity within soils around the reservoir.

\section{Establishing the parameters and the computing model for a local underground reservoir}

The function-simulation for a local underground reservoir comprises the following steps.

\subsection{Conceptual model. Functioning scenarios}

In order to establish the conceptual model concerning the flow issues for a local underground reservoir, have been identified the characteristics and the main problems for the considered groundwater layer, as well as for the 
irrigation system. There also have been established and structured the data needed for the modelling performed in the pre-processing stage.

\subsubsection{Data from the preliminary studies}

The primary data from field and/or lab studies, what must be considered for design of local sources for irrigation, mostly collected by specialized authorities, have to be systematized and analyzed on various categories: topographic, climatic, hydrologic, hydro-geologic, pedological, geological and chemical. Digital data can be efficiently be processed in EXCEL or/and MATLAB. Climatic, hydrologic and hydro-geologic data are to be statistically prepared (e.g. multi-annual averages).

3.1.1.1 Topographic data. This can be extracted (if there is no digital map available) from a physical support (maps, etc) on layers, by means of scanning and digitization with a special software, e.g. DIDGER 3. So, topographic data are to be prepared in a pre-processing format compatible with the digital processing software (e.g. FEFLOW v5.1, AQUA3D).

With an object-oriented approach, topographic data have been structured as objects in various classes, hence resulting a series of layers: $1^{\circ}$ the delimitation of the catching surface of the underground reservoir, $\mathrm{S}_{\mathrm{UB}}$, and of the culturable irrigable surface, $\mathrm{S}_{\mathrm{IS}} ; 2^{\circ}$ level curves; $3^{\circ}$ hydrographical network; $4^{\circ}$ distribution of pressures (observation drillings); $5^{\circ}$ shafts and exploitation drains; $6^{\circ}$ road network and towns; $7^{\circ}$ soils and textures classes and using classes. The outlines of the modelling domain have been established in order to create boundary conditions very similar to the natural feeding of the aquifer and reservoir's exploitation.

To analyse the possibility of an artificial recharge of the exploited aquifer, there must be known the relative position of the surfaces $S_{I S}$ and $S_{U B}$. To this purpose will be determined the surface $\mathrm{S}_{\mathrm{IS} \_\mathrm{UB}}=\mathrm{S}_{\mathrm{IS}} \cap \mathrm{S}_{\mathrm{UB}}$. Thereupon must be analysed the typical possible situations: $\mathrm{I}$ - the surfaces $\mathrm{S}_{\mathrm{IS}}$ and $\mathrm{S}_{\mathrm{UB}}$ are disjunctive if $\mathrm{S}_{\mathrm{IS} \_U B}=\Phi$; II - in the case $\mathrm{S}_{\mathrm{IS} \_ \text {UB }} \neq \Phi$, the surface $\mathrm{S}_{\mathrm{IS}}$ is included (or includes) partially/totally in surface $\mathrm{S}_{\mathrm{UB}}$, if $\mathrm{S}_{\mathrm{IS} \_\mathrm{UB}} \subseteq \mathrm{S}_{\mathrm{UB}}$ (or $\left.\mathrm{S}_{\mathrm{IS} \_\mathrm{UB}} \subseteq \mathrm{S}_{\mathrm{IS}}\right)$.

3.1.1.2 Climate data. This must be collected from several meteorological stations that influence the aquifer's hydrographic basin; these data being: $1^{\circ}$ monthly precipitations; $2^{\circ}$ monthly temperatures; $3^{\circ}$ potential and/or real evapo-transpiration (if these data are not available, the PET, the potential evapotranspiration, can be computed on basis of recorded temperatures, being preferably to use the Penman formula. The RET, the real evapo-transpiration can be taken as equal of the PET).

3.1.1.3 Hydrological data. These are to be collected when levels in the aquifer are significantly influenced by levels within one or two rivers (when the upper aquifer is located in rivers' inferior terraces). These data refer to the status of monthly levels and are to be collected from $1 . . .2$ hydrometric stations, preferably one in the upstream area and the other one in the downstream area. 
3.1.1.4 Hydro-geological data. These are to be collected from hydrogeological stations or from the hydroelectric plant's observation drillings and are comprising: $1^{\circ}$ the geometrical and geotechnical parameters (depth of basic impermeable layer, rocks' texture); $2^{\circ}$ the pressure levels in the observation drillings (measurements to be performed according to the frequency instructed by the station); $3^{\circ}$ pumping tests (useful for the assessment of conductivity/ transmissivity); $4^{\circ}$ the specific underground flow discharge.

3.1.1.5 Chemical data. These are needed in order to establish the water's quality parameters. Furthermore, these data will serve to define the type of uses for the water and to appreciate the risks of pollution.

\subsubsection{The basic work assumptions to the construction of the conceptual model}

Considering the fact that the aquifer is or of the alluvial type, or that it shows only a little thickness, the multi-layer structure has been assimilated to an onelayer aquifer, for which have been adopted equivalent values in vertical direction.

In such an underground medium the velocities of flow are relatively small, and the vertical distribution of pressures gets closer to the one imposed by hydrostatic law. This means that the vertical component of pressure gradient is small compared to the horizontal one. Besides, in case of non-isotropic aquifers, the vertical hydro-conductivity is much lesser than the horizontal one.

Therefore the modelling domain, $\Omega$, allows a two-dimensional (2D) representation, which is situated in the horizontal plane $(x O y)$. Within the $x O y$ plane the isotropy conditions are fulfilled. On the other hand the homogeneity conditions are fulfilled only in some zones, the domain being generally nonhomogeny.

So the values of the parameters (aquifer's characteristics: porosity, hydroconductivity, infiltrations, levels, flows) have imposed punctually or in certain zones (in characteristic points, respectively on elements of model's domain).

In the issue, for the considered model there have been adopted the following working assumptions: $1^{\circ}$ the aquifer is considered as a isotropic and saturated porous medium; $2^{\circ}$ homogeneity and isotropy conditions are fulfilled for a representative elementary volume $(\mathrm{REV})$ for a given study scale (Darcy law's applicability conditions); $3^{\circ}$ the aquifer storages a free water table; $4^{\circ}$ the flow process is assumed to take place in horizontal direction (Dupuit assumption); $5^{\circ}$ the aquifer's feeding is made by means of infiltrations in the modelling domain and by filtration flows on the domain's outlines (coming from aquifers located outside the modelling domain, having a higher hydrostatic level, or from a river).

Next to the data processing will be identified the main variables (hydraulic load) and the secondary variables (velocity, flows rates), concerning the global flowing within the aquifer. 
In the conceptual model must be considered the type of the adequate zone for the arrangement of the underground reservoir, as well as the technical solutions for the water catching and for the accomplishment and the operation of the irrigation system. So, for example, at the base of a terrace the groundwater will be retained by the implantation of a vertical screen that is constrained in the impermeable base of the aquifer, and for the water catching will be realized a pumping shaft with two horizontal drains, which are laid along the screen. The ground water provice (domain) of so accomplished underground reservoir is bounded as follows: $1^{\circ}$ at upper part - by the ground water line; $2^{\circ}$ at bottom - by the vertical impermeable screen equipped with drains and $3^{\circ}$ sidewise - by the greatest slope lines; beside these, it was adopted continuous flow irrigation but only in growing season.

The design of the underground reservoir was performed by soil water balance method for a water management year and this consists in two distinct stages: a) the dimensioning and $b$ ) the verification.

In the verification stage, the functioning scenarios for an underground reservoir differentiate between two distinctive work stages: $1^{\circ}$ the stage of reservoir filling (when no water is tapped from the collecting - tapping shaft); $2^{\circ}$ the stage of reservoir draining (when water is tapped from the collecting tapping shaft). So, starting from a known initial potential of the aquatic layer and taking into account both scenarios, which alternate in time, will be determined the evolution of the aquifer's potential (hydraulic head) for the analyzed period (for example a water management year).

The mathematical models for the two designing stages are following presented.

\subsection{The mathematical model for the dimensioning stage}

In this stage must determine the drain's lengths, $L_{1}$ and $L_{2}$, using the equation:

$$
\beta_{\mathrm{UR}} q_{\mathrm{UF}}\left(L_{1} \cos \alpha_{1}+L_{2} \cos \alpha_{2}\right) T_{\mathrm{WMY}}=\bar{M} A_{\mathrm{IS}}
$$

where:

$\beta_{\mathrm{UR}} \quad=$ storage coefficient of underground reservoir, [1];

$q_{\mathrm{UF}} \quad=$ specific underground flow discharge, $\left[\mathrm{L}^{2} \mathrm{~T}^{-1}\right]$;

$\alpha_{1}, \alpha_{2}=$ angles between underground flow's direction and normal to the drains' axis, [1];

$T_{\mathrm{WMY}}=$ time span of the water management year, [T];

$A_{\text {IS }}=$ culturable irrigable area, $\left[\mathrm{L}^{2}\right]$;

$\bar{M} \quad=$ the weighted mean irrigation rate, corresponding to the agricultural cultures' (cultures\#agr's) composition, [L].

The rate $\bar{M}$ was evaluated as follows.

$\bar{M}=\sum_{(j)} \chi_{j} M_{j}=\sum_{(j)} \frac{A_{j}}{A_{\mathrm{IS}}} M_{j}$, with $\sum_{(j)} \chi_{j}=1$ and $\sum_{(j)} A_{j}=A_{\mathrm{IS}}$, 
where:

$\chi_{j} \quad=$ the weight of the culture\#agr $j$ in the considered composition;

$A_{j} \quad=$ occupied area of the culture\#agr $j$ in the considered composition;

$M_{j} \quad=$ the irrigation rate of the culture\#agr $j$.

\subsection{The mathematical model for the verification stage}

In this stage the mathematical model is constituted by the equations which govern the flowing, as well as the initial and the boundary conditions - for essentially horizontal 2D flow through unconfined (phreatic) aquifer (Diersch [1]). These equations and conditions (vertically averaged/depthintegrated) were particularized in keeping with the working assumptions of the conceptual model.

\subsubsection{The governing equations}

These following: vertically averaged mass conservation (of the liquid phase) and depth-integrated Darcy flux:

$$
\begin{gathered}
S \frac{\partial h}{\partial t}+\frac{\partial \bar{q}_{x}}{\partial x}+\frac{\partial \bar{q}_{y}}{\partial y}=\bar{Q}_{p}, \\
\bar{q}_{x}=-\left(h-z^{\text {bottom }}\right) K \frac{\partial h}{\partial x} \text { and } \bar{q}_{y}=-\left(h-z^{\text {bottom }}\right) K \frac{\partial h}{\partial y},
\end{gathered}
$$

where:

$S \quad=$ storage coefficient, [1];

$h \quad=$ hydraulic head, [L];

$\bar{q}_{x}, \bar{q}_{y}=$ depth-integrated Darcy velocity along the coordinate axis, $\left[\mathrm{L}^{2} \mathrm{~T}^{-1}\right]$;

$\bar{Q}_{p} \quad=$ depth-integrated specific sink/source rate of liquid, $\left[\mathrm{LT}^{-1}\right]$;

$K \quad=$ hydraulic conductivity, $\left[\mathrm{LT}^{-1}\right]$;

$z^{\text {bottom }}=$ bottom geometry (height) of aquifer, [L].

Specific rate $\bar{Q}_{p}$ is represented by the infiltration water losses' rate, what has been computed on basis of following balance equation:

$$
\bar{Q}_{p}=\left\{\begin{array}{c}
\frac{1}{T_{\mathrm{GS}}}\left[(1-\sigma) \mathrm{PP}_{\mathrm{GS}}-\mathrm{RET}_{\mathrm{GS}}+\bar{M} \frac{A_{\mathrm{IS}} \mathrm{UB}}{A_{\mathrm{UB}}}\right], \text { for } t \in T_{\mathrm{GS}} ; \\
\frac{1}{T_{\mathrm{OGS}}}\left[(1-\sigma) \mathrm{PP}_{\mathrm{OGS}}-\mathrm{RET}_{\mathrm{OGS}}\right], \text { for } t \in T_{\mathrm{OGS}},
\end{array}\right.
$$

where:

$T_{\mathrm{GS}} \quad=$ time span of growing season, [T];

$T_{\mathrm{OGS}}=$ time span out of growing season, $T_{\mathrm{OGS}}=T_{\mathrm{WMY}}-T_{\mathrm{GS}}$, [T];

$\sigma \quad=$ flow coefficient, assessed in function of the terrain's slope and texture, and also the type of use; 
$\mathrm{PP}_{\mathrm{GS}}, \mathrm{RET}_{\mathrm{GS}}=$ precipitations, $\mathrm{RET}$ respectively, since growing season, [L]; $\mathrm{PP}_{\mathrm{OGS}}, \mathrm{RET}_{\mathrm{OGS}}=$ precipitations, RET respectively, since out growing season, [L];

\subsubsection{The initial conditions}

These conditions are:

$$
h(x, y, 0)=h_{I}(x, y) \text { with }(x, y) \in \Omega
$$

where $h_{I}$ is a known function, defined on all analysed domain, $\Omega$, which reflects the initial spatial distribution of the status variable $h$.

\subsubsection{The boundary conditions}

The conditions on the domain's outside, $\Gamma$, are only of integral Neumann type:

$$
K\left(\frac{\partial h}{\partial x} n_{x}+\frac{\partial h}{\partial y} n_{y}\right)=-\bar{q}_{h}^{R}(t) \text { with }(x, y) \in \Gamma,
$$

where:

$n_{x}, n_{y}=$ components of the normal unit vector (positive outward), [1];

$\bar{q}_{h}^{R}(t)=$ depth-integrated normal boundary fluid flux (positive outward), $\left[\mathrm{L}^{2} \mathrm{~T}^{-1}\right]$.

In the stage of reservoir drainage, only on the bottom domain's outside, $\Gamma_{2}$, we have:

$$
\bar{q}_{h}^{R}(t)=\frac{\bar{M} A_{\mathrm{IS}}}{T_{\mathrm{GS}}\left(L_{1} \cos \alpha_{1}+L_{2} \cos \alpha_{2}\right)}>0 ;
$$

but in the stage of reservoir filling, on all outside $\Gamma$, the conditions (7) became of natural type, id est $\bar{q}_{h}^{R}(t) \equiv 0$.

\section{Technology for the construction of a underground reservoir}

If we take into discussion (in order to diminish costs) only the tapping of upper aquifers, we have to keep in mind that the flow rate to such a catching (small depth) decreases in summertime when the feeding infiltrations become minimal, whilst the needs for irrigation are maximal. From this considerations results that the water supply of farms, which do not have access to surface waters, or for which the access is limited by huge pumping and water transport costs, is an issue which could be solved by means of: a) designing and building local reservoirs using less deep groundwater layers, such as the upper aquifer; b) conceiving a technology for the construction of water reservoirs at affordable cost.

Taking into account the fact that in Romania such irrigation systems have not yet been built and the prices for conventional technologies are prohibitive, at least today, we propose a new technology, based on a Patent [2], the invention being awarded with gold at World Fair Eureka-2000 in Belgium. 


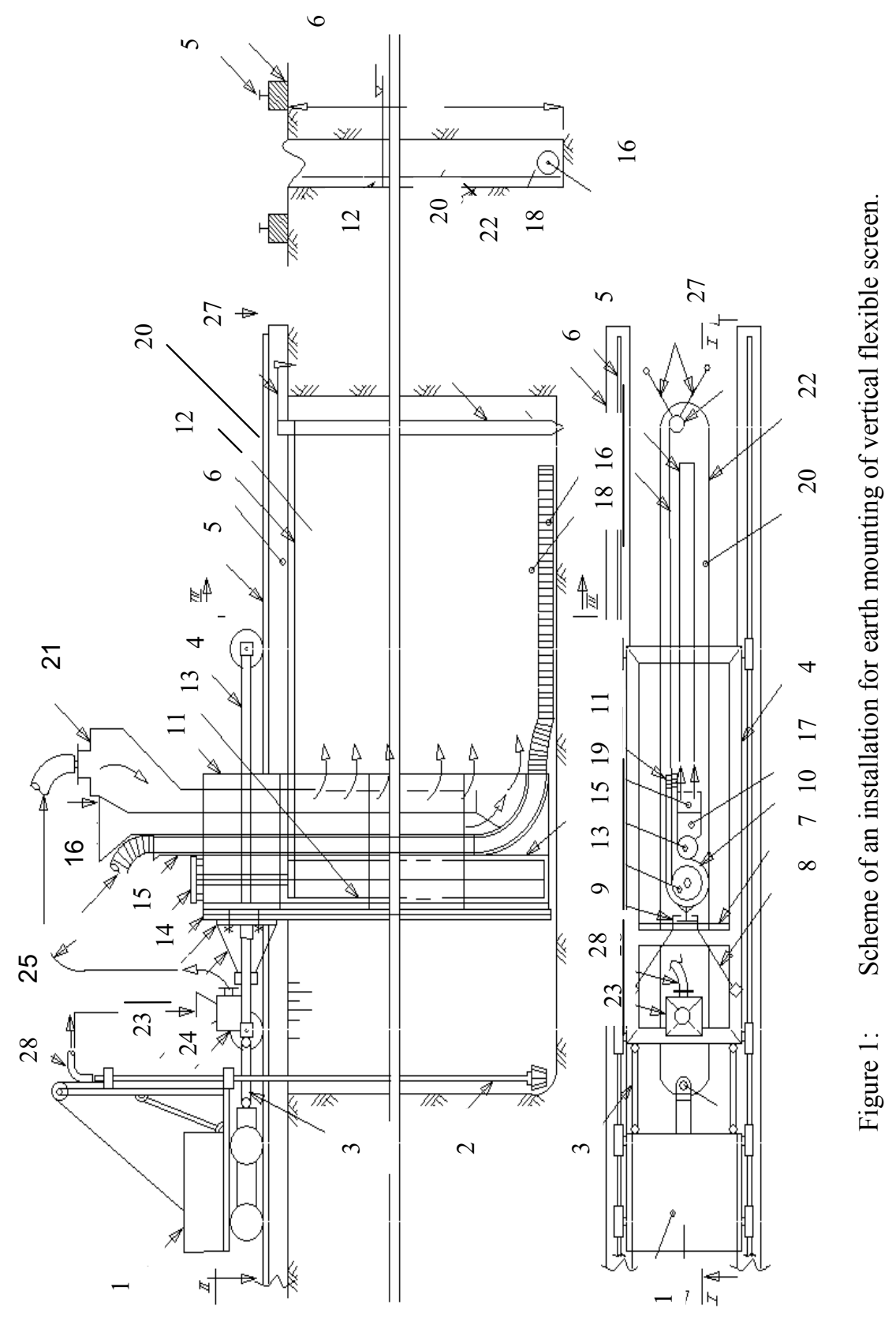


The installation, fig.1, allows the barring of groundwater flow with a vertical membrane-like impermeable screen (20), located in plane, and shaped as a "V", in order to intercept the current lines of the flowing water. The installation comprises a digger (1), the resulted residues being pumped towards a bunker (21) fitted with two discharge gutters (19) via several slots, back towards the trench, on each side of the membrane-screen, un-winded from a drum (13), which is vertically introduced in caisson (15) equipped at the posterior part with an exit slot of the membrane-screen (20) in the trench. The patent describes also how several screens can be connected, thus their length (that is the underground dam) being not limited.

The implementation of the technology means the following stages: a) when the screen is installed, a column for the pumping shaft will be mounted, the shaft being located in the angled peak of the screen; b) after finishing the screen, the installation has to be completed with a draining system along its wings. The draining lines, which are connected with the pumping shaft, are mandatory if the groundwater gets accumulated in rocks with low porosity; c) a depth drain is necessary to be executed, a drain able to be mounted from surface down to depths of 10-20 meters.

It is to be mentioned that the realization costs of the membrane-like flexible screen are ten times lower than that of conventional technologies (like readyparts screens; monolith concrete screens, earth and cement screens).

\section{Conclusions}

$1^{\circ}$ The critical global climate changes that also have an impact on Romania, imposed the extended use of groundwater in order to complete and balance the surface-waters, also providing a better protection of the environment.

$2^{\circ}$ The technology presented in this paper for the realization of underground reservoirs has to be mainly focused on the lowering of investment costs. On the other hand this becomes a crucial condition for a sustained development of agriculture.

$3^{\circ}$ The presented conceptual and mathematical models contribute to scientific substantiation of the underground reservoirs' for irrigation design.

\section{References}

[1] Diersch, H.J.G., FEFLOW finite element subsurface flow \& transport simulation system), Reference Manual, WASY Institute, Berlin, 2002.

[2] Nițescu E., Installation for the Execution of Vertical, Nonpermeable Screens, Romanian Patent RO- 94275, 1987.

[3] Niţescu E., Popescu Şt., Toma D., Chiorescu Esmeralda a.o., Technological Development Concerning Creation and Tapping of Ground Water for Irrigation, Grant tip A - CNCSIS, nr. 536/2005. 